\title{
Über die Gestaltung von Austauschbeziehungen
}

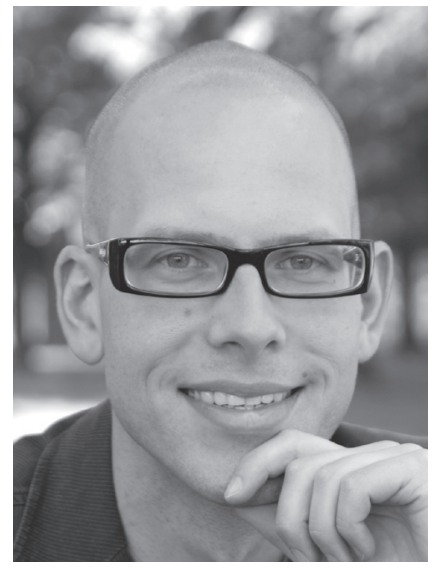

VON PAUL REINBACHER Dr. Paul Reinbacher lehrt und forscht nach Tätigkeiten in Industrie und Beratung derzeit am FH Campus Wien, insbesondere im Europäischen Joint Degree Masterprogramm Sozialwirtschaft und Soziale Arbeit (SOWOSEC). Aktuelle Interessen sind Wissen, Gewissen und Geschmack von Organisationen sowie ökonomische Aspekte sozialer Wechselwirkungen und Ästhetik im Marketing. paul.reinbacher@ fh-campuswien.ac.at

\author{
Unter Marketing wird allgemein ein kunden- und \\ wettbewerbsorientiertes Verhalten und Entscheiden \\ von Unternehmen verstanden. Marketing ist auch für \\ Verbände und Organisationen der Sozialwirtschaft \\ nützlich, da bereits in vielen Bereichen der Sozialen \\ Arbeit ein Preis- und Qualitätswettbewerb \\ herrscht und die im Marketing verwurzelte \\ Kundenorientierung den Klienten zugutekommt.
}

Nach wie vor gilt Philip Kotlers Diagnose, wonach Sozialmarketing »is still a mystery to most and misunderstood by many", was möglicherweise gleichermaßen Ursache und Folge der geradezu ärgerlichen Oberflächlichkeit von im deutschsprachigen Raum verfügbaren Texten zu diesem Themenbereich darstellt. Schon allein daher sind Georg Kortendiecks differenzierte Überlegungen zum »Marketing im Sozialen Bereich « zu begrüßen, zumal sie in der gewohnten, gleichermaßen anspruchsvollen wie ansprechenden Form präsentiert werden.

Prof. Dr. rer. pol. Georg Kortendieck, Jahrgang 1959, studierte Volkswirtschaftslehre und war nach einer Tätigkeit als wissenschaftlicher Assistent in Geschäftsführer- und Leitungstätigkeiten in Verbänden und Einrichtungen der kath. Erwachsenenbildung in Niedersachsen aktiv. Seit 2001 hat er eine Professur an der Fakultät Sozialwesen der Hochschule Ostfalia, Braunschweig/ Wolfenbüttel mit dem Lehrgebiet Betriebswirtschaft im Sozialen Bereich und den Aufgabenschwerpunkten Betriebswirtschaft, Marketing, Strategisches Management, Kostenrechnung und Controlling, Volkswirtschaftslehre sowie Personalwirtschaft und Mitarbeiterführung in sozialen Einrichtungen.

Bereits das Einführungskapitel seines neuen Buches über Marketing in der Sozialwirtschaft bemüht sich um eine Gegenstandsbestimmung jenseits der verbreiteten, jedoch kurzschlüssigen Abgrenzung zum traditionellen Marketing unter Rückgriff auf den Dienstleistungscharakter Sozialer Arbeit allein. Die sich an dieser Stelle sodann bietende Gelegenheit einer genaueren Herausarbeitung der Unterschiede zwischen sozialwirtschaftlicher und erwerbswirtschaftlicher Perspektive auf Unternehmensebene mit dem Angelpunkt eines Konzepts "sozialer Dienstleistungen « (als dem Gegenstand einer ökonomischen Perspektive auf Soziale Arbeit) kann im Rahmen eines einführenden Lehrbuches leider nicht ergriffen werden, scheint jedoch nichtsdestotrotz für zukünftige Studien lohnenswert.

Indem der vorliegende Text »Marketing « formal als Sozialtechnik zur Gestaltung von Austauschbeziehungen konzeptualisiert, kann er sich sodann unter anderem dem Koordinationsmechanismus des Marktes sowie den Rollen (bzw. den Rollen-Sets) von Kundin und Klientin, etwaig auch: Konsumentin (bzw. im Dienstleistungsfalle eher: Prosumentin) aus einem weiteren Blickwinkel nähern, und erst im Zuge dessen, die Idee einer nicht schlüssigen (äquivalenten) Austauschbeziehung einführen. In sozial- und wirtschaftswissenschaftli- 
cher Perspektive wirft dies Anschlussfragen wie beispielsweise jene nach alternativen Praktiken des Tauschens auf.

Ausführungen zur Marketing- und Marktforschung dürfen in einer Überblicksdarstellung des vorliegenden Typs nicht fehlen, sind jedoch aufgrund der Kürze besonders herausfordernd in puncto Begrifflichkeit und Selektivität der Themen. Insofern mag die hier getroffene Auswahl zwar dazu verführen, Datenbeschaffung und Informationsgewinnung weniger als das aus empirischsozialwissenschaftlicher Sicht erforderliche Gewicht beizumessen, was jedoch durch Rückgriff auf einschlägige Literatur zur Methodik und Methodologie einfacher zu kompensieren ist als der mit diesem Text unterstützte Transfer handlungsleitender Konzepte des Marketing.

$\mathrm{Ob}$ die Bündelung der Maßnahmen im Marketing-Mix einer Systematik mit vier oder mehr Abteilungen folgen sollte, ist zwar nicht von zentraler Bedeutung. Angesichts des durchwegs betonten Dienstleistungscharakters der im sozialen Bereich vermarkteten Angebote überrascht allerdings, dass Georg Kortendieck für die klassischen vier Bereiche (product, price, promotion, place) optiert - zumal sich auch hier wieder eine Chance böte, herauszuarbeiten, wodurch sich möglicherweise die Anwendung von Prinzipien des Marketinginstrumentariums im sozialen Bereich substanziell von jener in anderen Feldern unterscheiden könnte. In ähnlicher Weise sind ja nicht zuletzt mit Blick in den Werkzeugkasten der Strategieentwicklung konzeptiv-methodische Innovationen für die Sozialwirtschaft ein Desiderat.

Schwerer wiegt allerdings die in guter Tradition vollzogene Trennung zwischen einer strategischen und einer operativen Dimension bei gleichzeitiger, geradezu naturgesetzlichen Zuweisung bestimmter inhaltlicher Themen, wodurch die sowohl strategischen als auch operativen Aspekte von Gestaltungsobjekten wie Leistungen, Gegenleistungen, Vertriebskanälen, Kommunikationsaktivitäten, Marktbeobachtung etc. in den Hintergrund geraten. Dies sowie die weitgehende Aussparung von Fragen der Implementierung im Sinne eines Marketing-Management (und MarketingControlling) ist ein Wermutstropfen aus einerseits fachlich-akademischer, andererseits professionell-handlungsprakti-

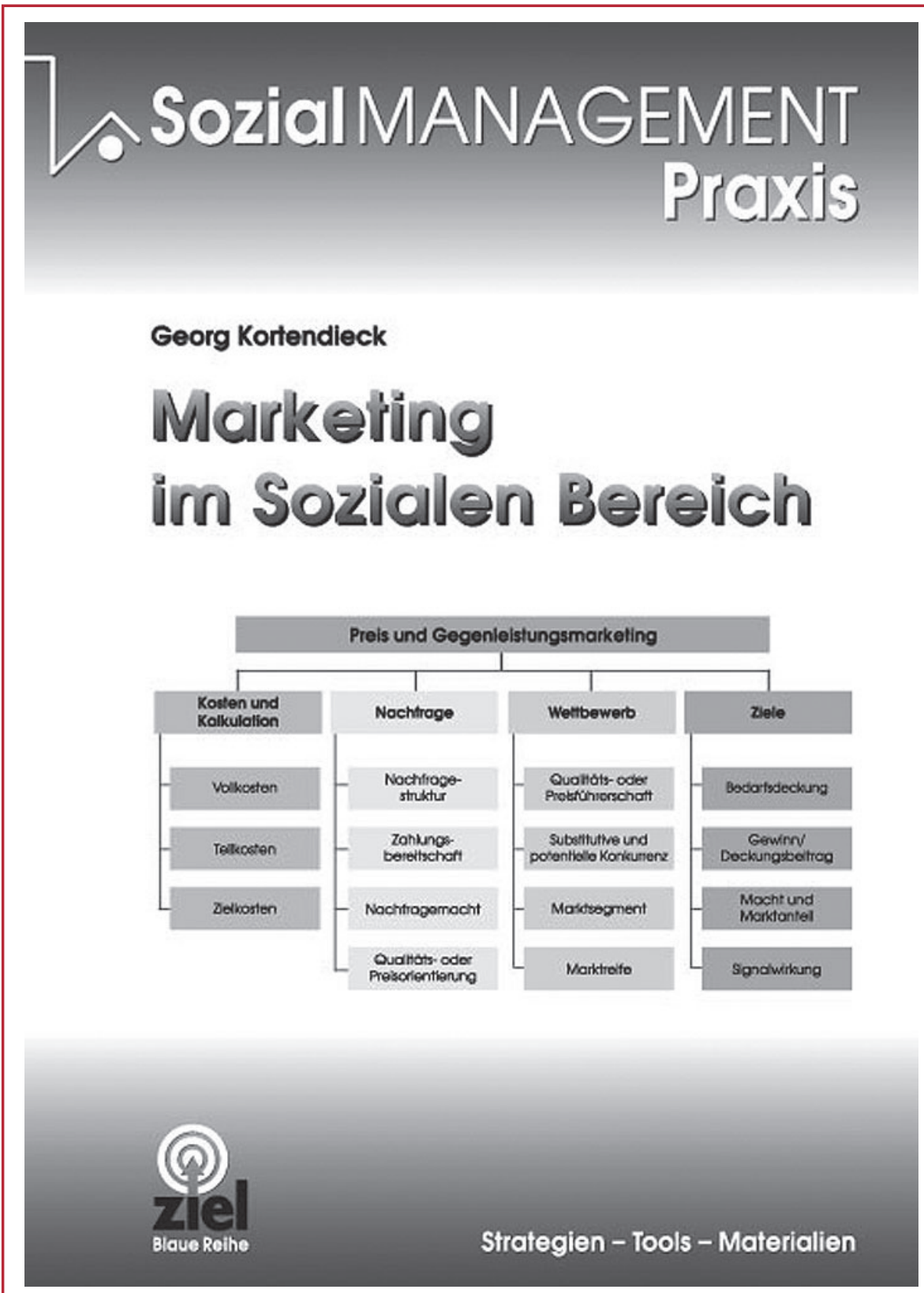

Georg Kortendieck: Marketing im Sozialen Bereich. Ziel Verlag, Augsburg: 2011. 168 Seiten. 25,80 Euro. ISBN 978-3-940562-60-9.

scher Sicht.

Dennoch ist der vorliegende Text aufgrund seiner Vorzüge wohl selbst die beste Empfehlung für all jene Studierenden und Berufstätigen, die einen kompakten und bekömmlichen Einstieg in die Thematik suchen (und die nicht zu sehr enttäuscht sind, wenn kaum prima facie einfache Checklisten, Standardrezepturen etc. geboten werden), sowie nicht zuletzt für jene Lehrenden, die sie bei diesem Einstieg unterstützen möchten. 